\title{
NONINVASIVE LOCALIZATION OF ACCESSORY PATHWAYS IN WOLFF-PARKINSON-WHITE SYNDROME BY TWO-DIMENSIONAL SPECKLE TRACKING ECHOCARDIOGRAPHY
}

\author{
By \\ Mohamed R. El-Bouhi, Mohamed Sami Abd El-Samea, Mansour M. \\ Aaref \\ Department of Cardiology, Faculty of Medicine, Al-Azhar University, Cairo, Egypt \\ Corresponding author: Mohamed Reda Mohamed Ahmed El-Bouhi \\ E-mail: muhammadelpohy938@gmail.com
}

\begin{abstract}
Background: Two-Dimensional Speckle Tracking Echocardiography (2D-STE) is a non-Doppler echocardiographic modality that allow quantification of myocardial deformity and its timing in 2D grey scale images, and was proved to be beneficial in assessment of dyssynchrony (STAR trial).

Objective: To investigate the capability of 2D-STE for localizing Accessory Pathways (APs) in patients with Wolf-Parkinson-White (WPW) Syndrome.

Patients and Methods: The prospective study included 15 patients with manifest AP indicated for invasive endocardial mapping and ablation. All patients were assessed by twelve-lead ECG, 2D-STE for the earliest activated site and invasive Electrophysiology study (EPs).
\end{abstract}

Results: Out of the 15 patients with left-sided APs, 2D-STE was able to accurately localize the site of AP in 10 patents (66.6\%), while electrocardiogram (ECG) was able to localize the site of AP in 12 patients (80\%).

Conclusion: 2D-STE has the ability to approximate the location of AP without the pitfalls of Doppler methods. However, ECG still more accurate and reproducible tool.

Keywords: Speckle Tracking Echocardiography, Accessory Pathway, Wolf-Parkinson-White Syndrome.

\section{INTRODUCTION}

The hallmark of Wolff-ParkinsonWhite (WPW) syndrome is early depolarization and thereby contraction of part of the ventricles at the site of atrioventricular APs (Delelis et al., 2012). In patients with Wolff-Parkinson-White (WPW) syndrome, ventricles are electrically and mechanically pre-excited through an accessory pathway, which cause eccentric ventricular activation and an asynchronous spread of ventricular depolarization (Park et al., 2013). LV wall motion abnormality was reported in patients with WPW syndrome, and some studies have suggested that presence of abnormal interventricular septal wall motion could be one of the causes of LV dyssynchrony and hence LV dysfunction that is reversible and not attributed to tachyarrhythmia (Dai et al., 2013). Whereas numerous body surface electrocardiogram algorithms have been described, the invasive electrophysiological study remains the 
gold standard method for localizing the site of the AP, hence allowing its catheter ablation (Delelis et al., 2012).

In myocardium with intact excitationcontraction coupling, electrical and mechanical events are tightly linked. Therefore, determining the site of first systolic motion is the basis of usage of different echocardiographic modalities in localization of accessory pathway (Cai et al., 2012). In the STAR study, the utility of speckle-tracking strain to quantify LV dyssynchrony was proven with the advantage of differentiating active motion from passive motion independent of Doppler angle (Tanaka et al., 2010). 2DSTE is a promising imaging modality which is reflected by the increasing number of publications focusing on its great potential clinical utility. Some have already heralded STE as 'the next revolution in echocardiography (Blessberger and Binder, 2010). 2D-STE modality is a non-Doppler method that allows the quantification of the myocardial deformation and its timing on $2 \mathrm{D}$ greyscale images without the potential pitfalls of Doppler-dependent methods (Delelis et al., 2012).

\section{PATIENTS AND METHODS}

Ethical approval was obtained from the medical ethical and research committee, Faculty of Medicine, Al-Azhar University. Written informed consent was obtained from each patient.

The study population consisted of 15 patients with manifest WPW syndrome, who were collected from the Cardiology Clinic of Al-Azhar University Hospitals, based on resting ECG findings, clinical history of frequent palpitation and/or documented ECG with attacks of atrioventricular reentrant tachycardia (AVRT). All enrolled patients were indicated for invasive endocardial mapping and radiofrequency catheter ablation (RFCA). The study was performed at Cardiology Department, Faculty of Medicine, Al-Azhar University between June 2019 and March 2020.

\section{Patient's selection:}

\section{Inclusion criteria:}

Patients with manifest WPW syndrome, with left-sided AP, who were indicated for invasive endocardial mapping and ablation.

\section{Exclusion criteria:}

Presence of structural heart disease such as coronary artery disease, congestive heart failure, valvular or congenital heart disease. Any rhythm other than sinus rhythm or AVRT. Presence of chronic systemic, inflammatory or neoplastic disease. Patients with right-sided AP as predicted from surface ECG was excluded after confirmation by endocardial mapping, due to unavailability of right ventricular speckle tracking echocardiography system.

The surface ECG of each subject was assessed to estimate the location of APs according to the criteria of Arruda algorithm (Arruda et al., 1998). Briefly, it is based on classifying the initial $20 \mathrm{~ms}$ of the delta wave in leads I, II, aVF and V1 as positive, negative or isoelectric, and assessment of the ratio of $\mathrm{R}$ and $\mathrm{S}$ wave amplitude in leads III and V1. Accordingly, the diagnostic flow chart of Arruda algorithm was followed to determine one of 10 possible locations 
around mitral and tricuspid annuli. Patients with right-sided AP were excluded after confirmation by EP study.

All subjects underwent a comprehensive transthoracic echocardiography before the planned invasive endocardial mapping using Philips Affinity 50 Ultrasound system with an S4-2 (2-4 MHz) cardiac sector transducer. With simultaneous ECG recording and over three cardiac cycles, all standard views were obtained including parasternal long-axis view, parasternal short-axis views (basal, mid and apical), apical 4-chamber view, apical 2-chamber view and apical long-axis view. All measurements were according to the guidelines of American Society of Echocardiography (ASE). The physician analyzer was unaware of clinical data or ECG data.

Standard echocardiographic measurements were obtained including aortic root diameter (AoD), left atrial diameter (LAD), left ventricular enddiastolic diameter (LVEDD), left ventricular end-systolic diameter (LVESD), interventricular septum thickness (IVST), and left ventricular posterior wall thickness (LVPWT). Left ventricular ejection fraction (LVEF) was assessed by modified Simpson method, while cardiac valves and diastolic function were assessed by a comprehensive Doppler study.

For left ventricular 2D-STE, the ECGgated 2D data over three cardiac cycles were stored and transferred to a computer for offline analysis. Six sectors were used including the three parasternal short axis views (basal, mid and apical) and the three aforementioned apical views. The images were analyzed for 2D-STE using 2D wall motion tracking software named Automated Cardiac Motion Quantification AI (aCMQAI). The automatic tracking of the myocardial contour on an end-systolic frame was carefully verified with manual correction if necessary, to ensure optimal tracking and to cover the entire thickness of LV myocardium. Using the time to peak tool in the aCMQAI software, the LV myocardial segmental deformational timing were computed and automatically represented in a single bull's eye depiction divided into 17 segments identical to the 17 segments ASE model (Cerqueira et al., 2002). The segment with the shortest estimated time represented the earliest activated site and hence the location of the AP.

All WPW patients underwent an invasive electrophysiological study for radiofrequency catheter AP ablation. The ablation procedure was considered successful if anterograde and retrograde conduction of the AP was completely abolished. This was associated with inability to induce AVRT. Endocardial mapping was considered the gold standard reference for localization the AP and was compared with the 2D-STE-derived location (Issa et al., 2019).

\section{Statistical analysis:}

Data were analyzed using Statistical Package for the Social Science (SPSS) version 20. Quantitative data were expressed as mean \pm standard deviation (SD). Qualitative data were expressed as frequency and percentage.

The following tests were done: The relationship between the ACP site determined by endometrial mapping, and either ECG or 2D-STE were determined 
by Cramer's V test which was based on 0.05 was considered significant.

Pearson's chi-squared statistics. P-value <

\section{RESULTS}

The study group included 15 patients, 7 males and 8 females. The mean age was $34.87 \pm 7.909$ years $($ range $=21-51$ years), while the Body Mass Index (BMI) mean was $24.20 \pm 1.935 \mathrm{Kg} / \mathrm{m}^{2}$ (range = $21-27 \mathrm{Kg} / \mathrm{m} 2$ ). Subject characteristics were summarized in Table (1).

Table (1): Subjects Characteristics

\begin{tabular}{|c|c|c|c|}
\hline \multicolumn{2}{|c|}{ Parameters } & \multicolumn{2}{c|}{$\begin{array}{c}\text { Patients } \\
(\mathbf{N}=15)\end{array}$} \\
\hline \multirow{2}{*}{ Age (years) } & Mean \pm SD & \multicolumn{2}{|c|}{$34.87 \pm 7.909$} \\
\cline { 2 - 4 } & Median & \multicolumn{2}{|c|}{36} \\
\hline \multirow{2}{*}{ Sex $($ No \& $\%)$} & Male & 7 & $46.7 \%$ \\
\cline { 2 - 4 } & Female & $\mathbf{8}$ & $\mathbf{5 3 . 3 \%}$ \\
\hline \multirow{2}{*}{ BMI $\left(\mathrm{kg} / \mathrm{m}^{2}\right)$} & Mean \pm SD & \multicolumn{2}{|c|}{$\mathbf{2 4 . 2 0 \pm 1 . 9 3 5}$} \\
\cline { 2 - 4 } & Median & \multicolumn{2}{|c|}{$\mathbf{2 4}$} \\
\hline
\end{tabular}

Table (2) demonstrated the data of all patients regarding localization of their APs, with comparison between the accurate site as derived from the EP study and the predicted sites from ECG Arruda algorithm and 2D-STE. It's obvious that many of ECG potential sites can cover two echo potential sites. The difference between the modalities regarding nomenclature was obvious. Therefore, agreement between modalities was not always reflected by the modality-specific names. 
Table (2): Comparison between ECG, 2d-STE and EP study regarding location of AP in each patient

\begin{tabular}{|c|c|c|c|c|c|}
\hline $\begin{array}{l}\text { WPW } \\
\text { Subjects }\end{array}$ & Sex & $\begin{array}{l}\text { Age } \\
\text { Years }\end{array}$ & $\begin{array}{l}\text { Prediction From } \\
\text { Arruda Algorithm }\end{array}$ & $\begin{array}{l}\text { Prediction From } \\
\text { 2D-STE }\end{array}$ & $\begin{array}{l}\text { EP study } \\
\text { (Gold Standard) }\end{array}$ \\
\hline 1 & $\mathrm{M}$ & 51 & LP/LPL & Infero-Lateral & LPL \\
\hline 2 & M & 21 & LL / LAL & Antero-Lateral & LAL \\
\hline 3 & $\mathrm{~F}$ & 43 & LPS & Inferior & LP \\
\hline 4 & $\mathrm{M}$ & 36 & RPS & Inferior & RPS \\
\hline 5 & $\mathrm{~F}$ & 28 & LL / LAL & Anterior & A \\
\hline 6 & $\mathrm{~F}$ & 26 & LPS & Infero-Septum & LPS \\
\hline 7 & $\mathrm{~F}$ & 29 & LL / LAL & Antero-Lateral & LAL \\
\hline 8 & M & 33 & $\mathrm{LL} / \mathrm{LAL}$ & Antero-Lateral & LAL \\
\hline 9 & $\mathrm{~F}$ & 39 & LP/LPL & Infero-Lateral & LPL \\
\hline 10 & M & 43 & LP/LPL & Infero-Lateral & LPL \\
\hline 11 & $\mathrm{~F}$ & 27 & LP/LPL & Antero-Lateral & LPL \\
\hline 12 & $\mathrm{~F}$ & 36 & LL / LAL & Infero-Septum & LPS \\
\hline 13 & $\mathrm{M}$ & 37 & $\mathrm{LL} / \mathrm{LAL}$ & Infero-Lateral & LAL \\
\hline 14 & $\mathrm{M}$ & 33 & LL / LAL & Antero-Lateral & A \\
\hline 15 & $\mathrm{~F}$ & 41 & LP/LPL & Infero-Septum & MS \\
\hline
\end{tabular}

A: anterior, AL: antero-lateral, IL: infero-lateral, I: inferior, IS: infero-septum, AS: antero-Septum, LAL: left antero-lateral, LPL: left postero-lateral, LP: left posterior, LPS: left postero-septum, RPS: right posteroseptum, MS: mid septum.

Perfect agreement was achieved in $10 / 15(66.6 \%)$ patients, with significant $P$ value $=0.038$, but all the remaining five patients demonstrated adjacent agreement on either side of the perfect agreement site (Figure 1). 


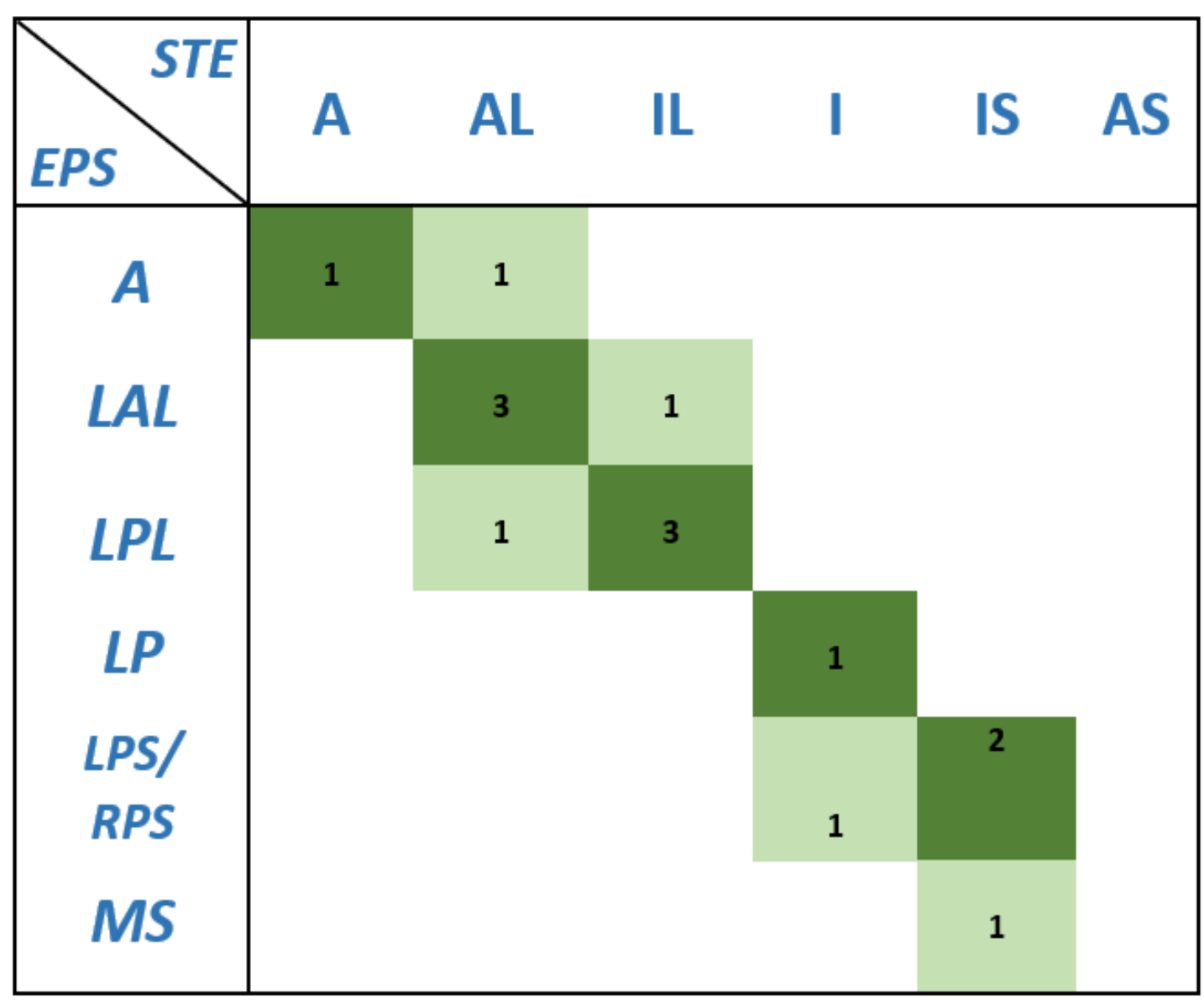

Figure (1): Correlation between predicted accessory pathway location by 2D- STE and the actual location based on EPS. Green cell indicates a perfect match between echo-predicted site and the EPS Localization site. pale green cell indicates Non-perfect but adjacent localization. Numbers indicate numbers of patients. A: anterior, AL: antero-lateral, IL: infero-lateral, I: inferior, IS: infero-septum, AS: antero-Septum, LAL: left antero-lateral, LPL: left postero-lateral, LP: left posterior, LPS: left postero-septum, RPS: right postero-septum, MS: mid septum.

Perfect agreement was achieved in $12 / 15(80 \%)$ of patients, with $\mathrm{P}$ value $=$ 0.001 , and adjacent agreement within a range on either side of the perfect agreement site was observed in one patient. The remaining two patients demonstrated disagreement (Figure 2). 


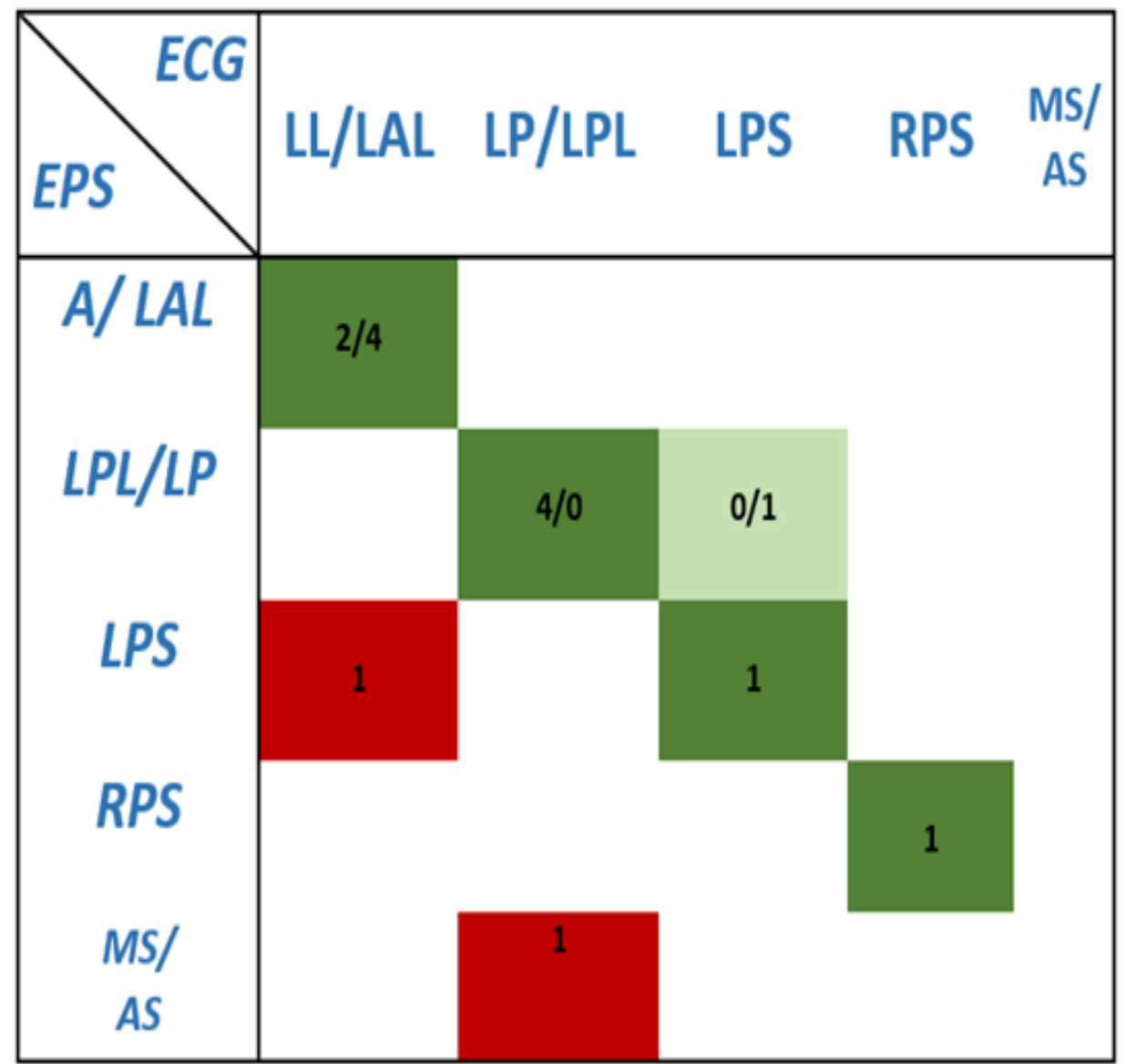

Figure (2): Correlation between predicted accessory pathway location by ECG and the actual location based on EPS. Green cell indicates a perfect match between echo-predicted site and the EPS Localization site. pale green cell indicates Non-perfect but adjacent localization. Red cell indicate disagreement. Numbers indicate numbers of patients.LL/LAL: leftlateral/left antero-lateral, LP/LPL: left posterior/left postero-lateral, LAL: left antero-lateral, LPL: left postero-lateral, LP: left posterior, LPS: left postero-septum, RPS: right postero-septum, MS: mid septum.

In comparison with EP study as the gold standard for localizing APs, ECG was superior to 2D-STE, $80 \%$ and $66.6 \%$ respectively. However adjacent agreement within a range on either side of the accurate site was in favor of the 2D-STE (Figure 3). 


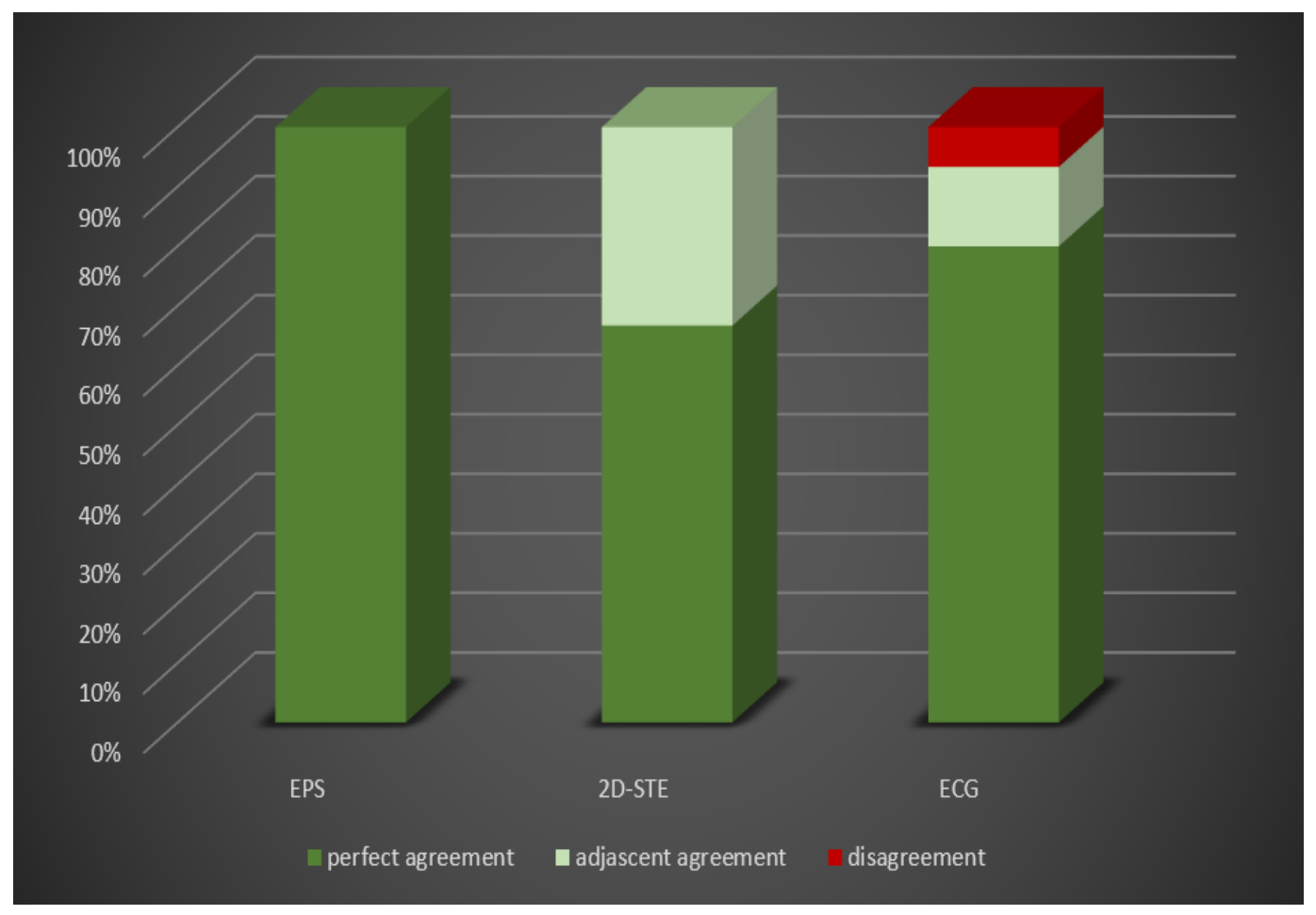

Figure (3): Comparison between 2D-STE and ECG regarding accuracy of AP localization with EP study as the gold standard

\section{DISCUSSION}

The present study demonstrated clear superiority of ECG over 2D-STE regarding approximation of the location of APs. However, the accuracy of ECG algorithms in recent studies has not reached the accuracy that was previously reported by their designers (Maden et al., 2015). In a study that tested seven different ECG algorithms in localizing APs, all algorithms were less accurate in predicting APs location than expected from data from their original authors (Wren et al., 2012). 3D-STE was found superior to ECG in localizing APs with regard that EP study as the gold standard (Ishizu et al., 2016). It was concordant with the results of Esmaeilzadeh et al. (2013) which showed superiority of strain imaging parameters over ECG prediction. Other echocardiographic conventional modalities including M-Mode and TransEsophageal Echocardiography (TEE) were inferior to ECG (Cai et al., 2012).

The capability of 2D-STE for localizing APs was confirmed in the present study. It is concordant with the results of Delelis et al. (2012) who concluded that 2D-STE has the ability to approximate the location of APs in addition to its ability to assess myocardial dyssynchrony. The 2D-STE potential abilities regarding APs location was confirmed by the study of Ishizu et al. (2016), a noninvasive isochrone activation imaging (AI) system with 3D-STE was developed which showed matching results with our study. Tissue Doppler (TD) myocardial imaging has higher accuracy (80-90\%) than conventional M-mode and 2-D imaging in localizing left-sided accessory pathways. However, it is still 
not ideal or right-sided pathways (Cai et al., 2012). However, the clinical utility of TDI-derived strain has been limited by artefacts caused by myocardial translational motion, requirement for optimal Doppler alignment, poor reproducibility, time consuming off-line analysis and angle dependency. In contrast, 2D-STE is a non-Doppler method that allows quantification of the myocardial deformation and its timing on 2D greyscale images without the potential pitfalls of tissue Doppler (Delelis et al., 2012).

\section{LIMITATIONS}

The number of patients was relatively small. The unavailability of right ventricular STE software excluded right sided AP cases. Echocardiograph has an inherent limitation of interpersonal as well as intrapersonal variations.

An important fundamental limitation was that 2D-STE dealt with mechanical activation and not electric activation. We assumed that electromechanical coupling is well preserved in the normal heart such that the mechanical activation pattern seems to reflect the electric phenomenon of the myocardium. Each electric activation is followed by an electromechanical one, that is, the depolarization of a cardiac muscle cell is followed by an uptake of calcium, which triggers contraction after a certain electromechanical delay of a few milliseconds.

The clinical role of noninvasive diagnosis of location of the AP with speckle-tracking may be limited, as it does not alter the therapeutic plan, and as ablation is based on the localization by the electrophysiological testing. However, the present findings in the setting of WPW syndrome, highlights the accuracy of speckle-tracking imaging in the assessment of pre-systolic contractile events and hence myocardial dyssynchrony.

\section{CONCLUSION}

In concordance with its proved utility in assessment of myocardia dyssynchrony, 2D-STE has the ability to approximate the location of AP without the pitfalls of Doppler methods. However, ECG still more accurate and reproducible tool. Practically, since invasive EP study is irreplaceable, either ECG or 2D-STE should be used as an orientation rather than precise localizing tools.

\section{REFERENCES}

1. Arruda, M. S., McCLELLAND, J. H., Wang, X., Beckman, K. J., Widman, L. E., Gonzalez, M. D. and Jackman, W. M. (1998): Development and validation of an ECG algorithm for identifying accessory pathway ablation site in Wolff-Parkinson-White syndrome. Journal of cardiovascular electrophysiology, 9(1): 2-12.

2. Blessberger, H. and Binder, T. (2010): Two-dimensional speckle tracking echocardiography: basic principles. Heart, 96(9): 716-722.

3. Cai, Q., Shuraih, M. and Nagueh, S. F. (2012): The use of echocardiography in Wolff-Parkinson-White syndrome. The international journal of cardiovascular imaging, 28(4): 725-734.

4. Cerqueira, M. D., Weissman, N. J., Dilsizian, V., Jacobs, A. K., Kaul, S. and Verani, M. S. (2002): Standardized myocardial segmentation and nomenclature for tomographic imaging of the heart: a statement for healthcare professionals from the Cardiac Imaging Committee of the Council on Clinical Cardiology of the American Heart Association. Circulation, 105(4): 539-542.

5. Dai, C. C., Guo, B. J., Li, W. X., Xiao, Y. Y., Jin, M., Han, L. and Dong, J. Z. (2013): Dyssynchronous ventricular contraction in Wolff-Parkinson-White syndrome: a risk factor for the development of dilated 


\section{MOHAMED R. EL-BOUHI et al.,}

cardiomyopathy. European journal of pediatrics, 172(11): 1491-1500.

6. Delelis, F., Lacroix, D., Richardson, M., Klug, D., Kouakam, C., Brigadeau, F. and Ennezat, P. V. (2012): Two-dimensional speckle-tracking echocardiography for atrioventricular accessory pathways persistent ventricular pre-excitation despite successful radiofrequency ablation. European Heart Journal-Cardiovascular Imaging, 13(10): 840-848.

7. Esmaeilzadeh, M., Omran, M. T. S., Maleki, M., Haghjoo, M., Noohi, F., Haghighi, Z. O. and Abkenar, H. B. (2013): Noninvasive localization of accessory pathways in patients with WolffParkinson-White syndrome: a strain imaging study. The Journal of Tehran University Heart Center, 8(2): 65-66.

8. Ishizu, T., Seo, Y., Igarashi, M., Sekiguchi, Y., Machino-Ohtsuka, T., Ogawa, K. and Aonuma, K. (2016): Noninvasive localization of accessory pathways in WolffParkinson-White syndrome by threedimensional speckle tracking echocardiography. Circulation: Cardiovascular Imaging, 9(6): e004532.

9. Issa, Z., Miller, J. M. and Zipes, D. P. (2019): Clinical Arrhythmology and Electrophysiology: A Companion to Braunwald's Heart Disease (3rd ed). Pbl. Philadelphia, PA: Elsevier, chap 18: pp 599676.
10. Maden, O., Balci, K. G., Selcuk, M. T., Balci, M. M., Açar, B., Unal, S. and Selcuk, H. (2015): Comparison of the accuracy of three algorithms in predicting accessory pathways among adult Wolff-ParkinsonWhite syndrome patients. Journal of Interventional Cardiac Electrophysiology, 44(3): 213-219.

11. Park, H. E., Chang, S. A., Kim, J. H., Oh, I. Y., Choi, E. K. and Oh, S. (2013): Left ventricular dyssynchrony in pre-excitation syndrome: effect of accessory pathway location and reversibility after ablation therapy. Heart and Vessels, 28(2): 199-207.

12. Tanaka, H., Nesser, H. J., Buck, T., Oyenuga, O., Janosi, R. A., Winter, S. and Gorcsan III, J. (2010): Dyssynchrony by speckle-tracking echocardiography and response to cardiac resynchronization therapy: results of the Speckle Tracking and Resynchronization (STAR) study. European heart journal, 31(14): 1690-1700.

13. Wren, C., Vogel, M., Lord, S., Abrams, D., Bourke, J., Rees, P. and Rosenthal, E. (2012): Accuracy of algorithms to predict accessory pathway location in children with Wolff-Parkinson-White syndrome. Heart, 98(3): 202-206. 


\section{تحديا مكان الضفيرة الكهربية الزائدة عن طريث إستخدام التتبع

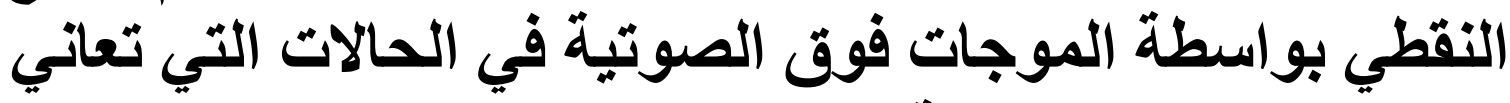

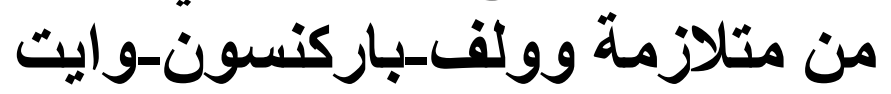
محمد رضا البوهي، محمد سامي عبد السميع، منصور مصطفي عارف قسم القلب، كلية الطب، جامعة الأزهر

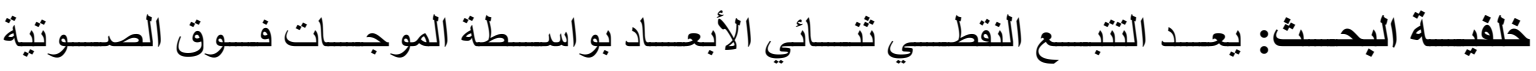

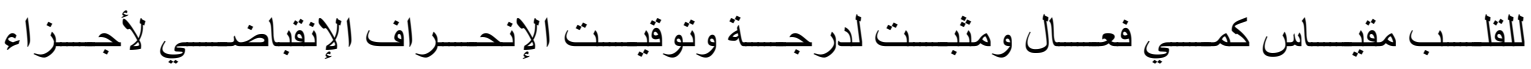
عضلة القلب.

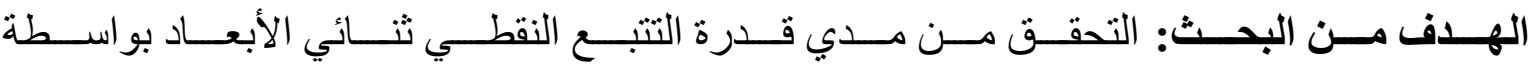

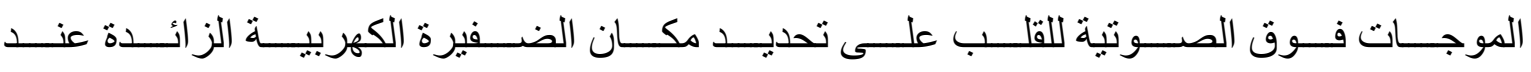
مرضي متلازمة وولف-باركنسون-و ايت.

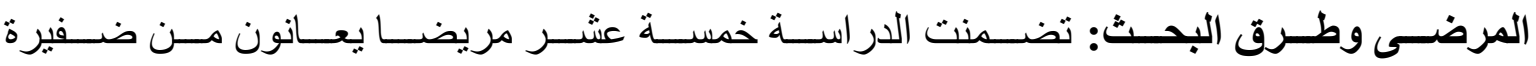

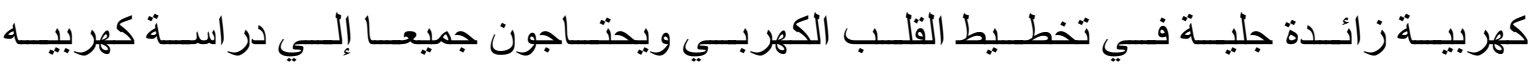

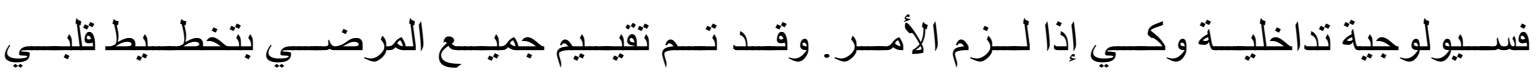

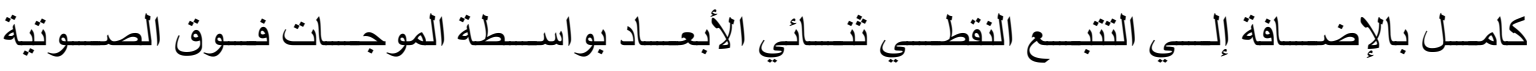
للقلب.

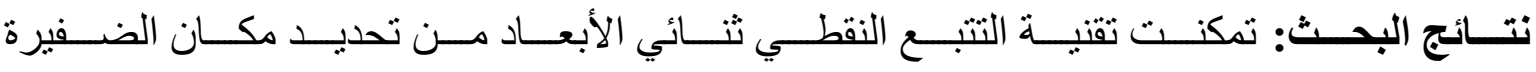

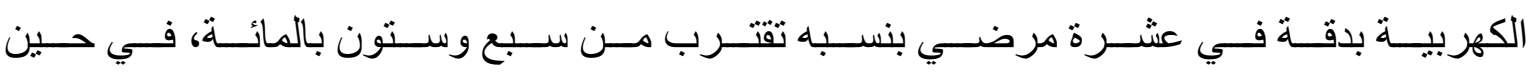

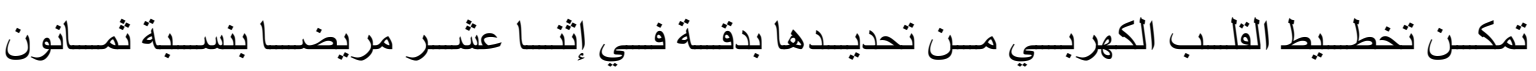
بالمائة.

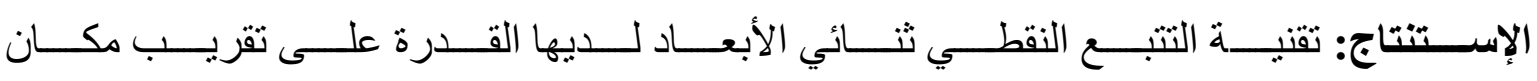

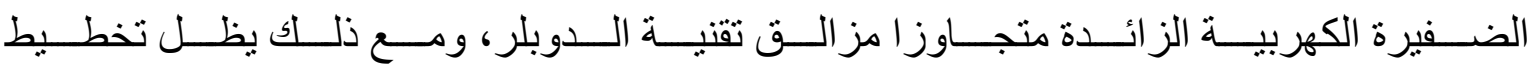
القلب الكهربي أداه أدق و أسهل. 